\title{
Commissioning MMS: Challenges and Lessons Learned
}

\author{
Paul Wood \\ Southwest Research Institute \\ 6220 Culebra Road \\ San Antonio, TX 78238 \\ 210-522-3275 \\ Paul.Wood@swri.org \\ Jennifer Reiter \\ Laboratory for Atmospheric and \\ Space Physics \\ 1234 Innovation Drive \\ Boulder, CO 80303 \\ 303-735-4410 \\ jennifer.reiter@lasp.colorado.edu \\ John Stone \\ Southwest Research Institute \\ 6220 Culebra Road \\ San Antonio, TX 78238 \\ 210-522-5073 \\ John.Stone@swri.org
}

\author{
Cheryl Gramling \\ NASA-Goddard Space Flight Center \\ Code 595, Greenbelt Road \\ Greenbelt Maryland 20771 \\ 301-286-8002 \\ cheryl.j.gramling@nasa.gov \\ Patrick Smith \\ Laboratory for Atmospheric and \\ Space Physics \\ 1234 Innovation Drive \\ Boulder, CO 80303 \\ 303-735-0921 \\ pat.smith@lasp.colorado.edu
}

\begin{abstract}
This paper discusses commissioning of NASA's Magnetospheric MultiScale (MMS) Mission. The mission includes four identical spacecraft with a large, complex set of instrumentation. The planning for and execution of commissioning for this mission is described. The paper concludes by discussing lessons learned.
\end{abstract}

\section{TABLE OF CONTENTS}

1. INTRODUCTION 1

2. COMMISSIONING BACKGROUND ........................... 2

3. CoMmissioning PLANNING................................. 4

4. COMMISSIONING EXECUTION .................................. 8

5. LESSONS LEARNED................................................. 11

6. CONCLUSIONS............................................................... 13

ACKNOWLEDGEMENTS .......................................... 14

REFERENCES...................................................... 14

BIOGRAPHY …............................................................ 15

\section{INTRODUCTION}

MMS [1] consists of a constellation of four (4) identical spin-stabilized spacecraft (S/C) with numerous instruments and processing modules making up the Solving Magnetospheric Acceleration, Reconnection, and Turbulence (SMART) Instrument Suite (IS). The S/C fly in a tetrahedral formation in highly elliptical orbits, necessitating precise maneuvers and onboard navigation. As the purpose of these S/C is scientific study, they are normally referred to as observatories, and, when we are referring to the entire satellite, we will use that term. The observatory is logically broken into two (2) pieces, the spacecraft bus, and the payload. We will discuss commissioning both.

MMS commissioning included many activities such as spacecraft bus activation, low-voltage turn on, deployments, and initial high-voltage activities; a shadow season with specialized commissioning activities; a post-shadow commissioning period including high-voltage activation, cross-calibration, and interference campaigns; and preparation for nominal science operations. In addition, each spacecraft performed thirty-two (32) maneuvers during the commissioning period.

Spacecraft bus commissioning included bus component activation, verification/characterization of the Radio Frequency (RF) communication, attitude control, onboard navigation, thermal control, and power systems, and included maneuvers to achieve mission orbit and attitude. Over the same time span, payload commissioning focused on initial activation and low voltage checkout of each sensor, boom deployments, gradual power up of high voltage instruments, and coordination of activities across the constellation. Almost all commissioning activities were completed at least four (4) times across the MMS constellation, although there were a few activities that were performed on a subset of observatories. Ground system commissioning included testing to identify and resolve network and multi-facility interface issues, which could not be tested prior to launch.

Planning for MMS commissioning addressed several critical activities and required coordination across multiple organizations, facilities, and resources including terrestrial 
and space communication networks. Mission planning responsibilities were performed by four (4) groups: (1) MMS program planners, responsible for building the long term (strategic) and day to day (tactical) schedule for payload commissioning coordinated with spacecraft activities, maneuvers, and ground contacts; (2) the Mission Operations Center (MOC), responsible for developing the tools necessary for S/C deck operations; the (3) Payload Operations Center (POC), responsible for planning the details of activities to be performed on the payload during each contact; and (4) Instrument Team Facilities (ITFs), responsible for providing instrument and investigation specific activity plans and instructions.

As the commissioning process proceeded from planning to execution, several challenges developed. The baseline commissioning plan quickly grew in complexity prior to launch as the amount of effort to commission four (4) spacecraft, the strict sequencing of operations to avoid instrument operational conflicts, and other factors had to be accommodated by the plan. Once under way, the need to adapt the schedule to deal with anomalies and investigate science targets of opportunity, the uncertainties posed by the limitations of ground emulation hardware, and delayed delivery of some instrument inputs began to require more instrument concurrent operations and last minute scheduling. However, thanks to solid preparation, commissioning was able to proceed with minimal impact from these potential disruptions.

\section{COMMISSIONING BACKGROUND}

\section{The Science}

The MMS Mission is an international, multi-institutional effort funded by NASA under the Solar-Terrestrial Probes program to "enable an understanding of magnetic reconnection in the boundary layers of the Earth's magnetosphere, which is formed by its interaction with the solar wind." [1] Magnetic reconnection occurs when the magnetic fields embedded in collision less plasmas become interconnected, converting magnetic field energy into the kinetic energy of the plasma ions and electrons [2]. In order to determine that magnetic reconnection is occurring, concurrent, three-dimensional maps of particle distribution functions, electric and magnetic fields, and plasma waves need to be made [3]. These measurement requirements flow directly into the mission configuration. The requirement for concurrent, three-dimensional (3D) measurements at varying temporal scales drives the use of a four (4) spacecraft tetrahedron; and the requirement for particle, and fields and waves measurements drives the instrument configuration of the observatories. Observations are separated into two (2) distinct science phases. Phase 1a observations occur on the dayside with crossings into the magnetosheath, while Phase 2 observations will occur in the magnetotail. A commissioning phase (Phase 0) occurred prior to Phase 1a, and a transitional phase (Phase 2a) between Phases 1a and $2 b$.
Each MMS observatory orbits in a $1.2 \times 12_{\mathrm{Re}}$ orbit for Phase 1 while maintaining a minimum quality tetrahedron throughout the science Region of Interest (ROI). Tetrahedron quality is based on the six (6) tetrahedron side lengths and the total volume within the tetrahedron. The tetrahedron is re-sized (ranging from approximately 160 to $10 \mathrm{~km}$ spacing for Phase 1, 400 to $25 \mathrm{~km}$ for Phase 2b) and re-constituted numerous times throughout the mission requiring precise locational knowledge.

During the operational phases, MMS collects highresolution data in the science ROI where reconnection is likely to occur, and lower resolution data outside the ROI. Within the ROI, medium resolution "survey" data are automatically downlinked for evaluation. Data are automatically tagged with quality measures, but humans perform a Scientist in the Loop (SITL) evaluation and ultimately determine what high-resolution data from a particular ROI will be downlinked. "Burst" activity data (the highest resolution data) not selected for downlink are automatically marked available for reuse by this process; "burst" activity data downlinked are automatically marked for reuse once they have been successfully downlinked.

\section{The Observatories}

Each MMS observatory is functionally identical. Attitude determination and control is provided by a complement of the Danish Technical University's four (4)-head star sensor and an Adcole Digital Sun Sensor, supplemented with ground-based attitude determination software. Due to the timelines for formation maneuver execution and the requirement on interspacecraft time knowledge, MMS flies an onboard orbit determination system, supplied by Navigator, a specialized Global Positioning System (GPS) receiver with weak signal acquisition, a high-resolution and accuracy accelerometer from Zin Technologies, and the Goddard Enhanced Onboard Navigation System (GEONS), an extended Kalman filter-based orbit estimator. A complement of twelve (12)-thrusters (banks of four (4)-lbf radial thrusters on two side panels and two (2) 1-lbf axial thrusters on the top and bottom decks) execute both attitude and orbit maneuvers. Maneuver control can be executed open loop or via an onboard delta- $\mathrm{V}$ or delta-H controller developed for MMS to enable execution of precision maneuvers on a spinning spacecraft with flexible body motion. The spacecraft bus was built in-house at NASA's Goddard Space Flight Center (GSFC).

The observatories include separate "S/C" and payload decks. The S/C deck (Figure 1) includes the power; communications; thermal management; S/C processor; solid state recorder; and guidance, navigation and control subsystems. The S/C deck also includes the payload's magnetic booms and one of the Energetic Particle Detector (EPD) components. Communications modes include high data rate communications to the Deep Space Network (DSN), and lower data rate to the Near Earth Network (NEN) and Space Network (SN), with all contacts at Sband. 


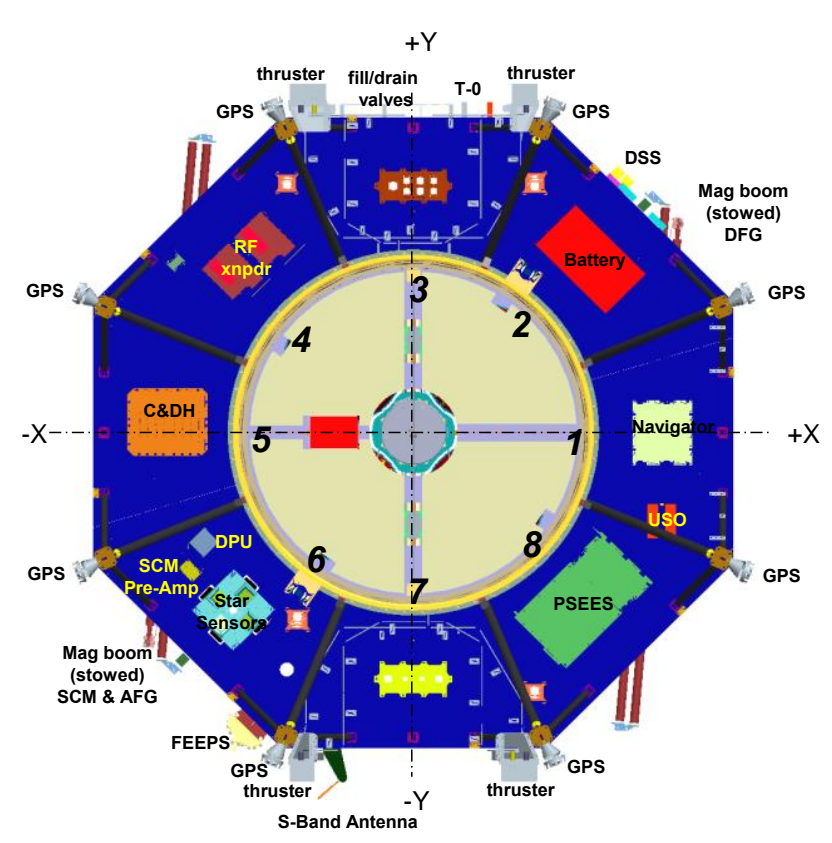

Figure 1. S/C Deck

The payload deck (Figure 2) contains the remainder of the instruments needed to make the required measurements. Each MMS payload deck contains six (6) major subsystems including instruments, observatory electrical potential management, and data processing/recording components. The six major subsystems are referred to as "investigations", several of which consist of multiple instruments, processing units, or other components. The investigations are: (1) Fields Suite - 3D electric and magnetic field sensors; (2) Fast Plasma Investigation (FPI) - full sky, thirty-two (32) energy step electron and ion sensors $(10 \mathrm{eV}-30 \mathrm{keV})$; (3) Energetic Particle Detectors (EPD) - all-sky ion and electron energetic particle sensors (20-500 keV); (4) Hot Plasma Composition Analyzer (HPCA) - 3D ion energy distributions of $\mathrm{H}^{+}, \mathrm{He}^{++}, \mathrm{He}^{+}, \mathrm{O}^{+}$, and background measurement; (5) Active Spacecraft Potential Controller (ASPOC) - to keep the observatory electrical potential within $4 \mathrm{~V}$ of the ambient environment, enabling consistency of measurements; and (6) Central Instrument Data Processor (CIDP) - controls S/C access to payload and stores science data for future analysis [4].

More importantly from a commissioning perspective, a total of twenty-six (26) payload components (per S/C) required commissioning activities as follows: Fields (four spin-plane double probes [SDPs], two (2) Axial Double Probes [ADP] with two (2) receiving elements, two (2) Electron Drift Instruments [EDIs], an Analog Flux Gate [AFG] Magnetometer, a Digital Flux Gate Magnetometer [DFG], and a Search Coil Magnetometer [SCM]), FPI (four Dual Electron Spectrometers [DES], and four Dual Ion Sensors [DIS]), and an Instrument Data Processing Unit (IDPU), EPD (two Fly's Eye Energetic Particles Sensors [FEEPS] and an Energetic Ion Spectrometer [EIS]), ASPOC (two (2) each with four (4) emitters), HPCA (1), and CIDP (1). The Fields investigation receiving elements, SDP, and ADP required one (1) or more deployment step prior to reaching the operational state.

\section{The Commissioning Team}

The subsystems were commissioned by teams comprised of personnel from two (2) continents, five (5) states, and five (5) time zones. Early commissioning activity (Low Voltage [LV] check out, High Voltage [HV] check out of one (1)

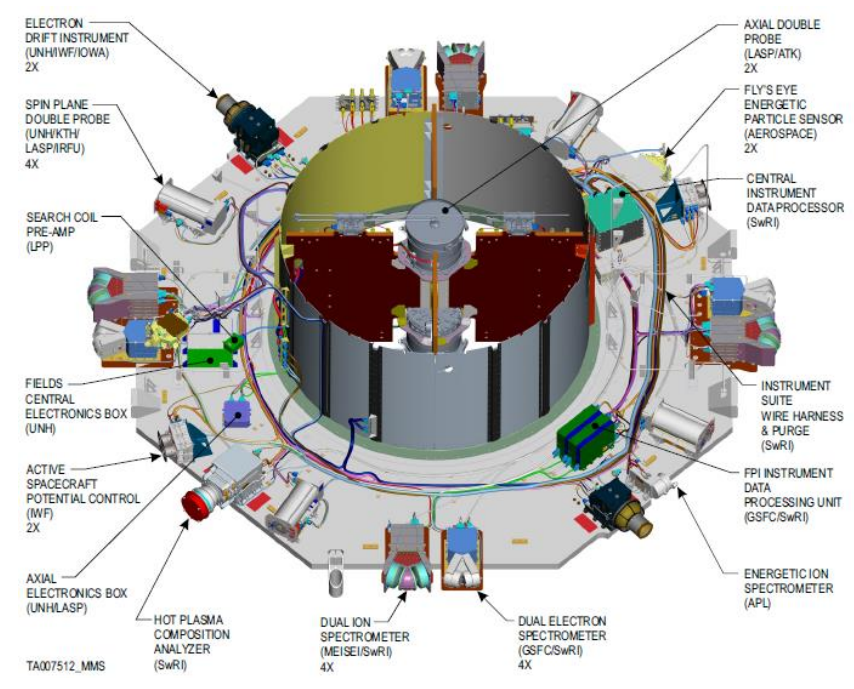

Figure 2. Payload Deck

instrument, and deployments) were performed on-site at University of Colorado/Laboratory for Atmospheric and Space Physics (CU/LASP), but later activities were coordinated remotely. The team consisted of:

- NASA Goddard - Mission Management, S/C Integrator, Mission Planning, MOC, Flight Dynamics Operations Area (FDOA), and the FPI ITF

- CU/LASP - Payload Operations Center (POC), Daily Planning and Payload Commissioning Execution

- Southwest Research Institute - IS integrator, IS Systems Engineers (ISSE), CIDP ITF, and HPCA ITF

- University of New Hampshire - Fields ITF including EDI and Fields deployables

- Johns Hopkins University/Applied Physics Laboratory - EIS ITF

- $\quad$ Aerospace Corp. - FEEPS ITF

- Institut für Weltraumforschung of the Austrian Academy of Sciences - ASPOC ITF

\section{Commissioning Roles}

MMS defined the following roles for commissioning: 
- Executive Planner - Coordinate across all the stakeholders (Project Management, Guidance, Navigation, and Control [GNC], Propulsion, Thermal, Power, RF Communications, Command \& Data Handling, POC, ITFs, and the three communication networks) to establish a master schedule that incorporates $\mathrm{S} / \mathrm{C}$ activities (such a maneuvers and bus calibrations) and IS activities.

- Flight Director (FD) - Overall S/C operation, determines when payload commanding is permitted within a communications contact.

- Product Development Lead (PDL) - Subject matter experts for the S/C subsystems. They monitor responses and provide $\mathrm{GO} / \mathrm{NO} \mathrm{GO}$ direction to the FD as activities are performed.

- $\quad$ POC Planner - Assimilate master schedule information and produce individual activity plans that account for $\mathrm{S} / \mathrm{C}$ and IS state, constraints (e.g., orbital location), and commanding requested by ITFs.

- $\quad$ POC FD - Overall responsibility for the IS with a focus on safely and efficiently completing the scheduled activities and responding to anomalies.

- $\quad$ POC Flight Controller (FC) - Voice of the POC to coordinate between the MOC and the ITFs, the FC follows the scheduled activities scripts.

- POC Command Controller (CC) - Execute the commands and scripts that control the activities under direction of the POC FC \& POC FD.

- $\quad$ ITF - Subject matter experts for the instruments. They monitor instrument responses and provide GO/NO GO direction to the POC as activities are performed.

- IS Systems Engineer (ISSEs) - Represent integrated IS concerns and (usually) act as subject matter experts for the CIDP. For critical activities such as deployments, ISSEs provide GO/NO GO direction for the CIDP ITF and the IS as a whole.

\section{Commissioning Planning}

\section{Commissioning Constraints}

With its large complement of instrument sensors, precision maneuvers, and four observatories, MMS proved unique among its spacecraft peers. Early on the commissioning team sought lessons learned from other multi-spacecraft missions. Cluster, operated by the European Space Operations Center (ESOC) in Darmstadt, Germany, offered insights into the human resource limitations and specifics about MMS predecessor instruments flying on Cluster. In addition, ESOC offered insights into their methods for planning maneuvers. Experience from the Time History of Events and Macroscale Interactions during Substorms
(THEMIS) and the Van Allen Probes (formerly known as the Radiation Belt Storm Probes [RBSP]) was sought and found to be minimally applicable, as neither mission had as many sensors as each MMS observatory, nor the need to execute precision maneuvers to achieve the correct attitude and orbital placement.

Based on the feedback from Cluster and experience from operating other spacecraft from GSFC, the following list of scheduling rules were instituted to mitigate the impact of commissioning on the human element, maintain safe instrument operations, and work within cost constraints. In the following lists, an (S) represents a soft constraint that could be broken if needed; an $(\mathrm{H})$ represents a hard constraint that involved mission safety; and a $(\sim)$ represents a hard constraint depending on the ITF team-attributes. Human factors constraints included:

- Plan the entire schedule for six (6) days ON/ one (1) day OFF per week to allow for one (1) contingency day per week (S)

- Keep similar activities for an investigation in the same relative time slots each day, and if more than one (1) event needs to be executed, keep them in same twelve (12)-hour shift to maintain human element. (S)

- If the time slot for a set of activities for an investigation has to change to a different shift, allow at least two (2) days between shift changes to allow human factors adjustment to time change. $(\sim)$

- Schedule no more than four (4) consecutive days on the same activity to avoid team burn-out; if one must perform an activity set for longer than four (4) days, the schedule should allow for an extra day off before restarting activities with that group of personnel. (S)

- Follow a 1-1-2 pattern for each new activity to give the ITF time to work any anomalies or quirks that arise. [Notionally, this translates to: perform the activity on $\mathrm{S} / \mathrm{C} \# 1$; wait a day then perform same activity on $\mathrm{S} / \mathrm{C}$ $\# 2$; wait a day, then perform the activity on S/C \#3 \& \#4 on the last day. (S)]

Cost constraints on the mission led to requirements for minimal network scheduling changes and consolidated travel periods for the ITFs during commissioning. The onboard navigation system needed a calibration period and would not be used operationally until after completing all the deployments, which led to a need for early orbit radiometric tracking data from the contacts with the networks. The S/C communication system supported only Doppler from DSN and NEN, and both range and Doppler from the SN. However, DSN provided higher data rate and was schedulable around apogee in the science Region of Interest. In addition, the flight dynamics team needed two (2) perigee transits between maneuvers to estimate the orbit in order to plan the next delta-V maneuver. All of these contributors led to the following additional set of rules: 
- Develop patterned contact schedules (see description below) to avoid the cost of ad hoc contact schedules. (H)

- Consolidate Activities for an investigation as close as possible in calendar days, given the instrument constraints and human factors rules, to minimize travel costs. $(\mathrm{H})$

- Restrict IS activations to DSN contacts to obtain higher real time telemetry rate. $(\mathrm{H})$

- Allow adequate time to downlink data via DSN on each spacecraft every day, at least one (1) hour per spacecraft (solved with contact patterns) $(\mathrm{H})$.

- $\quad$ Throughout the maneuvering period prior to GEONS calibration schedule a round-robin sequence of shorterduration SN contacts throughout the four (4) hours before and after perigee to obtain radiometric tracking in the most dynamic region of the orbit. $(\mathrm{H})$

- $\quad$ Separate consecutive large delta-V maneuvers on the same S/C by at least forty-eight (48) hours prior to GEONS calibration. $(\mathrm{H})$

The DSN contact pattern needed to fulfill several parameters. It had to be identified and implemented by specific rules for use by the multi-mission network scheduler, such as the time duration following perigee to start contacts. The patterns also had to provide flexibility for the activity planning and had to limit late-breaking changes to the contact schedule in case of a contingency. The patterned contact schedule used the first three weeks consisted of a round-robin of 2-hour contacts on each observatory starting with MMS-2, centering the four observatories around apogee in reverse numerical order to meet maneuver requirements for the perigee-raising campaign. The contacts changed to an A-day/B-day pattern starting at week four in order to:

- Provide four (4)-hour focus contacts on two observatories each day

- Provide adequate contact time on each observatory for data downlink

- Provide geometric diversity in locations over two consecutive days to accomplish radiometric tracking considerations

Besides the human factors and contact limitations already mentioned, the remaining constraints for execution fell into six categories:

1. Earliest time after launch to activate a sensor (to allow for outgassing);

2. Orbital location and attitude spin axis pointing and spin rate;
3. Operation exclusion window around shadows (power limits);

4. Operation exclusion window around maneuvers (to avoid plasma contamination);

5. Interval and frequency of an activity, and the duration after completing the previous activity before starting the next activity;

6. S/C environmental conditions, such as thermal and electrical potential, required to begin an activity.

\section{Communication}

To enhance communication among all the stakeholders, there was a clear early need to establish a Commissioning Working Group (WG) consisting of a member from each ITF knowledgeable about the instrument operations, the ISSEs, Mission Systems Engineers, and the commissioning planning team. The WG held planning telecons every fortnight in order to gather inputs on the commissioning operations for each sensor, to answer questions related to operations and resolve issues, and to educate the science team on the imposed constraints such as:

- Real time telemetry allocation

- Data delays for the different telemetry virtual channels

- Implications of the S/C lunar and solar eclipses

In addition, the Commissioning team held splinter discussions at each semi-annual Science Working Team meeting to discuss items of general interest to the whole audience and specific items that benefit from face-to-face interchanges, such as the nuances associated with sensitive high voltage operations.

For commissioning execution, the WG morphed into two meetings: a daily Tactical Planning meeting and a weekly Strategic Planning meeting. The Tactical meeting covered the upcoming three to five days with participation from the ISSEs, POC, MOC, FDOA, as well as any interested ITF. The Strategic Planning meeting covered a sliding multiweek period to focus on longer-range planning and special topics, with additional participation from the ITFs and S/C PDLs.

\section{Commissioning Procedure Development}

While the Integration and Test effort offered a preliminary view of the procedures needed to activate the instruments, most procedures migrated from I\&T needed significant changes for commissioning. In addition, each Activity for each sensor had a set of constraints that were either mandatory for instrument safety or highly desired in order to achieve the best results. The Commissioning WG defined a template for Activity Definition Forms (ADF) to provide descriptions of the activation and deployment activities as well as configuration updates and configuration and software loads. The ADF documented constraints and outlined steps for command and telemetry evaluation and included the following: 
1. the duration of each step,

2. the frequency of execution,

3. the commands and telemetry, and

4. the instrument end state with an indication of whether the instrument could be safely operated via ATS around maneuvers and shadows after procedure completion.

The ADFs served as input to Colorado System Test and Operations Language (CSTOL) [5] procedure development performed by both the ITFs and the POC. If an ITF delivered the CSTOL procedure, then the POC performed the syntax verification only; otherwise, the POC defined the CSTOL and performed the syntax verification. Each procedure was tested on the IS FlatSat prior to use operationally. Figure 3 shows a flow diagram for the CSTOL development process. A similar process was followed with the S/C subsystems: each S/C PDL provided inputs in a template to describe the activities needed to commission their subsystem with associated duration, commands, telemetry, and other parameters. The mission operations team developed System Test and Operations Language (STOL) procedures from these templates and validated them on the S/C Flatsat or the software-based. Mission Training Simulator prior to execution. Information for the IS activities were documented on the IS Commissioning Plan Wiki maintained by the POC.

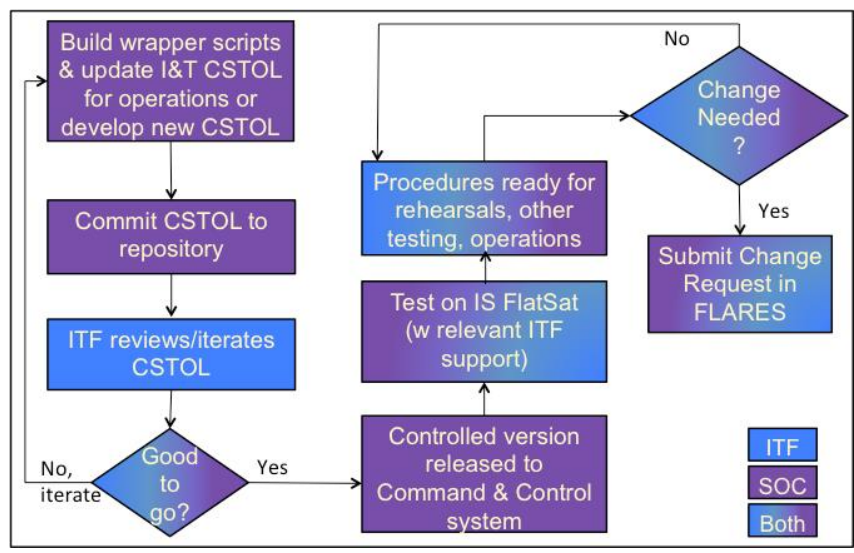

Figure 3. Process flow for developing CSTOL procedures for MMS commissioning

\section{Commissioning Activities}

Among the IS and the SC, there were eight hundred and forty-eight (848) activities defined prior to launch including an initial flight software load for EDI, and CIDP command sequence loads that repeated several times during commissioning. Table 1summarizes the number of activities per sensor and per $\mathrm{S} / \mathrm{C}$ as well as the total estimated duration needed to execute the procedures. Table 2 and Table 3 list the activity categories and number of unique activities for the IS and $\mathrm{S} / \mathrm{C}$, respectively. These activities were repeated on each observatory, and for many, they needed to be repeated at different intervals or after achieving certain observatory configurations.

Table 1. Pre-Launch Commissioning Activity Summary

\begin{tabular}{|c|c|c|c|}
\hline & \# of Activities & Total Duration & Realtime Duration \\
\hline CIDP & 36 & $31: 20: 00$ & $31: 20: 00$ \\
\hline ASPOC & 128 & 0:00:00 & $200: 23: 20$ \\
\hline EIS & 36 & 44:56:00 & 59:08:00 \\
\hline FEEPS & 20 & 17:04:00 & $12: 00: 00$ \\
\hline FIELDS & 107 & 279:29:00 & $215: 20: 00$ \\
\hline FIELDS/EDI & 164 & 152:00:00 & $152: 00: 00$ \\
\hline FPI & 78 & 154:30:00 & $106: 30: 00$ \\
\hline HPCA & 42 & 104:18:00 & $103: 33: 00$ \\
\hline IS & 13 & 130:00:00 & $130: 00: 00$ \\
\hline SC & 224 & $831: 38: 00$ & $84: 18: 00$ \\
\hline TOTAL & 848 & 1745:15:00 & 1094:32:20 \\
\hline SC \#1 & 207 & 441:04:30 & $220: 02: 20$ \\
\hline SC \#2 & 207 & $442: 54: 30$ & $223: 52: 20$ \\
\hline SC \#3 & $205^{\circ}$ & 438:54:30 & 219:52:20 \\
\hline SC \#4 & 205 & 438:54:30 & 219:52:20 \\
\hline SC-ALL & 24 & $323: 47: 00$ & $130: 45: 00$ \\
\hline
\end{tabular}

Table 2. Instrument Suite Commissioning Activity Categories

\begin{tabular}{|l|l|}
\hline Activity Type & Number \\
\hline Low Voltage (LV) & 16 \\
\hline High Voltage (HV) & 64 \\
\hline Deployments & 14 \\
\hline Instrument Suite Level (IS) & 13 \\
\hline Care and Feeding & 18 \\
\hline
\end{tabular}

Table 3. Spacecraft Commissioning Activity Categories

\begin{tabular}{|l|l|}
\hline Activity Type & Number \\
\hline Powering the CIDP and IS & 6 \\
\hline $\begin{array}{l}\text { Table and Real Time Sequence } \\
\text { Loads }\end{array}$ & 5 \\
\hline Oscillator and Clock Configuration & 3 \\
\hline Navigation Hardware and Software & 4 \\
\hline ACS Sensors \& Calibration & 9 \\
\hline RF Communications & 13 \\
\hline Thermal \& Power & 10 \\
\hline
\end{tabular}

\section{Planning Process}

The overall flow for the commissioning plan was based on WG expertise combined with the information in the populated templates.

- The first three (3) weeks after launch focused on S/C commissioning and IS LV commissioning, as well as 
the early mission maneuvers to raise perigee, deploy the magnetometer booms, and achieve the mission science attitude spin axis target.

- $\quad$ The following four (4) weeks focused on the SDP and ADP boom deployments and associated spin adjustment maneuvers, and initial $\mathrm{HV}$ activation.

- The energy balance constraints mandated a five (5)week shadow season, which limited the availability of instruments to those that needed the heat from their operation to survive the eclipses. A primary goal was to complete all deployments of booms and to bring up one of each sensor on at least one S/C prior to the shadow season to allow the science team time to evaluate data from all of the sensors.

- HV operations continued after shadow season along with maneuvers to achieve the tetrahedral formation at the initial scale size of $160 \mathrm{~km}$.

- After completing all HV Activation, the focus shifted to the interference and cross-calibration campaigns, followed by SITL, the Burst Data Management system, and observatory preparations for operations.

Commissioning had to complete prior to achieving the orbital orientation for commencing Phase 1 science operations (apogee vector at 1800 Geocentric Solar Ecliptic time), which occurred September 1, 2015, based on the nominal launch date.

Initially, a spreadsheet was developed to plan the activities for each day with color-coding used to identify the different observatories and types of activities. Each MMS S/C was assigned a color during I\&T that carried over into operations (MMS-1: yellow, MMS-2: blue, MMS-3: green, MMS-4: purple). Similarly, colors were assigned to different types of activities: S/C: orange; maneuvers: maroon; deployments: green; LV: blue; HV: red; shadows: dark gray; meetings: light blue. A notional identification of the DSN and SN contacts, the significant orbital locations (apogee, ROI entry, etc.), and the time from perigee provided a template onto which each activity was placed at the approximate time for execution. The scheduling process started by placing all of the shadows and maneuvers into the timeline as events. After the events, the activities were laid in according to their constraints, with ordering as described above.

The schedule determined how quickly commissioning could be executed. The benefit of this exercise was that it provided a useful indicator to the science team of the critical path leading to Phase 1 operations, helped to illustrate the complexity of the planning process, and set expectations for the level of effort needed during Phase 0. A favorable outcome was that the science team re-evaluated their initial inputs for activities and constraints and in many cases restructured their activities into reduced durations. Fitting all of these updated activities into patterned contacts proved to be a particularly useful approach. While it provided a realizable flexibility to readily identify specific times for rescheduling an activity in the future, it also lengthened the overall duration of Phase 0 . Figure 4 a shows a sample day of the spreadsheet for Orbit 4.

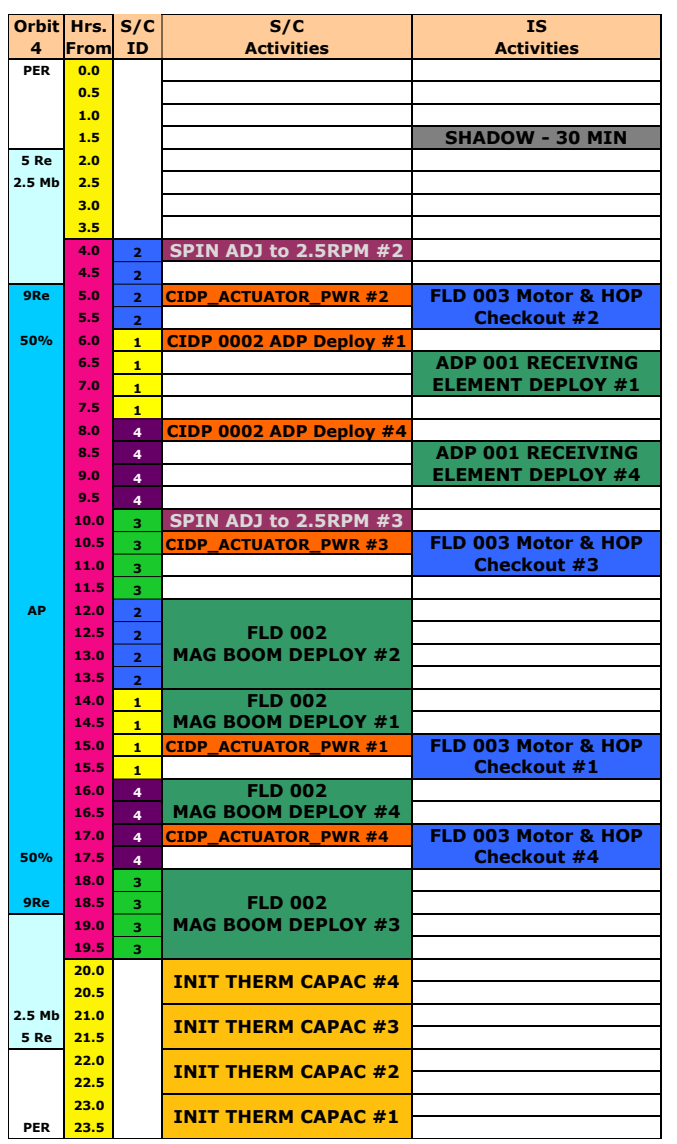

\section{Figure 4a. Orbit 4 Example from Commissioning Master} Plan Spreadsheet

There is great risk in maintaining a 'set' of all the different activities for each spacecraft and trying to update the whole schedule without error when the waterfall effect of an anomaly or even a missed contact disrupts the plan. While it may be possible to accomplish these types of rapid operational updates, the process is ripe for mistakes given that the collection of constraints for all of the activities cannot be easily enforced in a spreadsheet program. The conclusion was that a better scheduling tool was needed to act as a constraints warehouse capable of checking for constraint violations within the schedule. The team Activity Control Tool (tACT), developed by Hammers Company for previous missions at GSFC, was updated for MMS to handle multiple spacecraft. tACT ingests an Events file containing the network contact times and orbital events for each spacecraft and provides a means of defining an Activity and applying constraints based on any of the ingested Events or other Activities defined in the tool. The user develops a Gantt chart-like view using JavaScript Object Notation (JSON) language to define the attributes. The previously established color-coding was carried over 
into tACT for consistency in providing a visual interpretation for the MMS community. Figure $4 \mathrm{~b}$ shows the tACT complement to the Figure 4 a spreadsheet for Orbit 4.

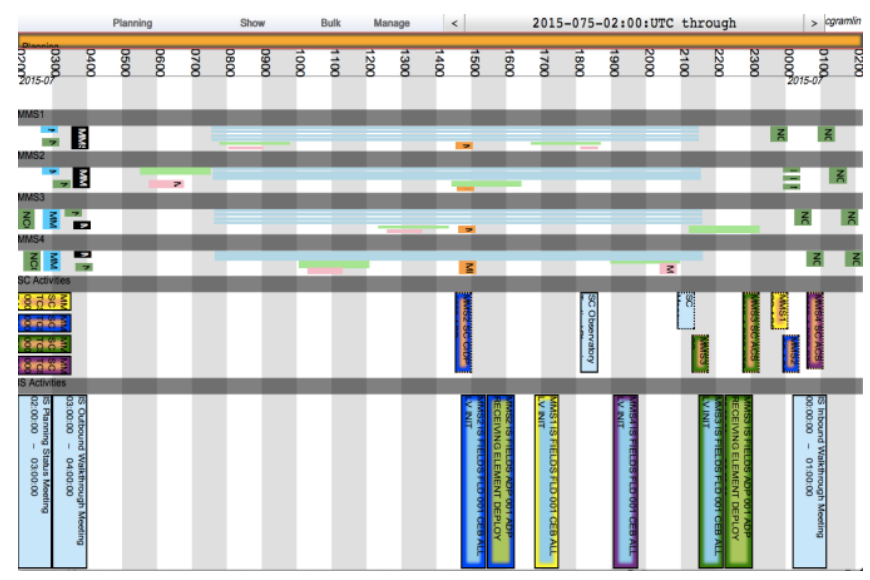

Figure 4b. Orbit 4 Example from tACT Plan

\section{COMMISSIONING EXECUTION}

\section{Preparation for Commissioning}

In addition the planning described in Section 3, several other types of preparation were conducted. These are described below.

Over the course of I\&T, the POC participation was slowly incorporated into the testing. For example, each observatory completed a lengthy thermal/vacuum test. During each such test, a Mission Rehearsal Test (MRT) that integrated POC commanding in place of the $I \& T$ commanding systems was conducted. This test provided an opportunity for the POC to exercise its systems and to practice operational procedures such as mode changes, validate interconnects, command and telemetry databases, and other necessary infrastructure.

Far in advance of launch, each ITF generated activity definitions. As mentioned previously, associated with each activity were one or more CSTOL scripts. These scripts were used to encapsulate and make routine the execution of the activity. Each script would be executed on the POC IS FlatSat for validation. Minimally, the scripts were verified to load successfully and be free of syntax errors. Most operational aspects could also be checked as the IS FlatSat includes relatively high fidelity instrument simulators. Some aspects of the execution cannot be thoroughly confirmed due to limitation in the IS FlatSat. For example, the IS FlatSat has no deployables, so although the switches in the CIDP that activate the deployables can be exercised, no feedback indicating the success/failure or progress of a deployment will be received.

In January 2015, only a couple of months prior to launch, a detailed training session was conducted at LASP where the ITFs, POC, MOC, and scientists were able to learn about instrument subsystems other than their own, along with POC and MOC expectations and needs so that a unified commissioning team could be established. This was a critical and extremely effective interchange that resulted in a shared view of the commissioning process among stakeholders. In addition, participants were able to develop mental models of observatory operation that were very helpful for thinking about subsystem interactions and preventing potential interferences or conflicts in commissioning planning and execution.

\section{Commissioning Day in the Life}

Each MMS Phase 0 orbit had duration slightly less than 24 hours. The high data rate DSN contacts occurred in the 8hour windows inbound and outbound near apogee. Most IS commissioning operations were generally not compatible with the higher radiation environment in the periapsis region. Thus, the 8-hour period around perigee focused on $\mathrm{SN}$ contacts for S/C operations, health and safety, and radiometric tracking data.

Each contact would typically be dedicated to a single investigation, although occasionally shorter activities for multiple investigations were able to be grouped together within a single contact. Activities were combined more as commissioning progressed and full instrument suite testing was performed for interference and cross-calibration efforts.

Ultimately, careful preparation and solid coordination between the mission planners, POC, ITFs, and ISSEs proved vital to maintaining such a tight schedule. Once the master schedule had been fed into tACT it needed to be broken into manageable parts that would allow the operations team to prepare for a given activity or set of activities. On a near daily basis, the tACT schedule was extracted and converted into an activity plan that laid out the detailed schedule for the next 14 orbits. The activity plan combined a graphical representation of each orbit with ground contacts, shadows, L-shell crossings, etc. overlaid as well as a summary of the activities to be executed on each DSN contact. This was provided to the IS commissioning team and served as a simple and effective tool to communicate the commissioning plan to the ITFs, ISSEs, and POC commissioning team.

With a clear view of the near-term activities, the POC planning team would begin assembling the CSTOL scripts and ensuring that everything was ready to execute on a given contact. This usually started with revalidation of the CSTOL scripts against the IS FlatSat in a more flight-like configuration. Some scripts were well vetted prior to launch and were rerun only for familiarity for the console teams. Others were developed much closer to launch or evolved as commissioning proceeded and these required careful testing and often updating to ensure they were ready for the flight assets. Additionally, as commissioning progressed and each observatory moved closer to its nominal operations configuration, some of the "standard" activities needed modifications or were found to no longer be valid. All of 
this made pre-execution FlatSat testing a necessary and meaningful exercise.

While having an activity definition, CSTOL scripts, and a scheduled DSN contact were necessary prerequisites, validating that a particular activity was ready for execution required putting those pieces together in context. The MMS Real Time Request (RTR) web-based form was the repository for capturing all the information that could impact a planned activity during the specific contact for which it was scheduled. RTRs consisted of:

- Purpose of the activity

- Applicable flight rules

- Notifications to be communicated to the MOC

- CSTOL execution steps

- Stored commands with the potential to impact the activity

- Scheduled contact information

- Contingencies

- ITF/ISSE/SOC approvals

- Operator execution notes

This form gathered all the information applicable to a commissioning activity in one place, allowing the POC planning team to verify that the activity could be executed as planned. While tACT provided orbital, environmental and scheduling constraint checking, the tACT tool is not able to check what is executing from stored command sequences or what other instruments might be in an active state during a planned activity. The POC planners were responsible for verifying those types of restrictions and communicated that verification in the RTR. The RTR also summarized how to carry out any planned contingencies for an activity, listing script names or commands the operators might need to send in the event of a failed deployment or anomalous behavior during an instrument checkout.

Once all testing and constraint validation was complete, the RTR would be reviewed by the ITF, ISSE and POC FD. The teams would vet the execution plan for an activity and provide notes, additional contingencies, clarifications, etc. as necessary. Once all parties were comfortable that an RTR had captured all the necessary details they provided an approval signature, confirming that they were in agreement the finalized plan for a given activity.

As previously mentioned, the sheer size of the commissioning team, as well as the geographic distance between the teams" "home bases" presented coordination challenges from the start. In order to minimize these challenges during phase 0 the POC established a planning cycle that allowed time for all parties to review and approve RTRs in advance of the planned DSN contact. RTRs were generated on a 4-orbit cycle, as outlined in Figure 5.

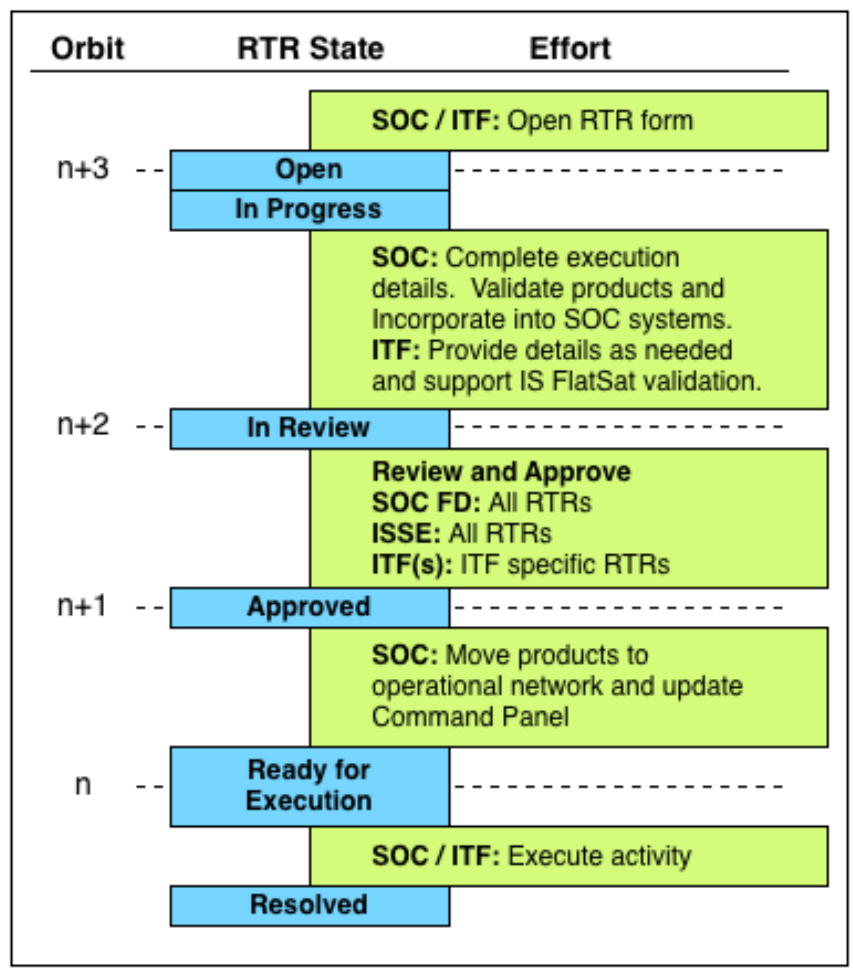

Figure 5. RTR Process

RTR status was reviewed each day at the POC planning meeting. RTRs for the next three orbits would have been generated by this point and would be in varying stages of completion as determined by the days remaining to execution. Activities planned for the upcoming orbit would be approved by the end of the planning meeting. Questions about pending RTRs would also be addressed, including timelines on CSTOL script delivery, altered contingencies or execution instructions as a result of instrument anomalies, or simply clarification on a given section of an activity.

The POC daily planning meeting served as the main status meeting for the IS commissioning team and was the prime forum for communication between the ITFs and the POC. The MOC \& Mission Planning team routinely participated as well, providing the entire group with a great deal of insight into spacecraft activities that might impact the commissioning timeline. Having one centralized meeting where all parties had a voice made it much easier to inform ITFs of potential impacts to their commissioning schedule due to anomalies or to coordinate multi-instrument activities. It also gave the POC a chance to check in with all the ITFs at the same time about script development, activity definitions, or planned anomaly troubleshooting.

One of the major challenges faced by the POC was establishing a working knowledge of a commissioning activity in order to execute it. With the large number of deployments, command-intense software loads, and other 
complex commissioning activities, it was crucial that the team performing a given activity be familiar with both its nominal flow and how to identify and react to problems that may arise. To that end, the first hour of each shift was spent walking through the activities planned for the coming 12 hours. These walkthroughs were led by the POC Planner and benefited from the participation of the on-shift ITF, ISSE, POC FD, FC, and CC. At a minimum, walkthroughs reviewed:

1. The CSTOL script or scripts to be executed

2. Possible contingencies and responses

3. Any limit violations expected during the activity

4. Any timing constraints or restrictions due to stored commands executing during the contact

5. The order activities should be executed on a contact if there was more than one

Holding these walkthroughs once per shift easily brought the on-console team up to speed without overburdening them with learning the minutiae of every planned commissioning activity.

Boom deployments were deemed critical activities and as such highly detailed walkthroughs were held for these. In the walkthroughs, the ITFs presented technical background on the activity and contingency flowcharts were reviewed extensively so that the whole team knew where the potential stopping points were. The MOC operators also participated in these walkthroughs as there was often a good deal of interaction between the MOC and the POC planned for the activity. These detailed walkthroughs were held a few days in advance when all of the crew members who would execute them on each observatory could participate. This also allowed the FC and CC who would be on console the time to run through the CSTOL scripts on the flatsat before they did it in flight. Having the entire team participate in these walkthroughs was extremely beneficial and this training made the entire team more knowledgeable about the activity.

\section{Transition to Nominal Ops}

A key factor was the incremental method used to ease the way into operations. Prior to entering the shadow season at the end of the second month of commissioning, an FPI activity to test an operational Orbit in the Life (OITL) (FPI.010.2) served as the initial segue into operations,. This was the first activity necessitating each ITF to consider other instrument's states and configurations and the first point at which multiple IS activities were coordinated using the S/C Absolute Time Sequence (ATS) mechanism. Participation was limited, since each sensor had not been fully activated and calibrated at that time. After executing FPI.010.2 the first time on MMS3, a target of opportunity arose during the shadow season when MMS aligned with the Van Allen Probes (VAP); this again brought the multidiscipline IS team together to execute for common science goals. Given the power limitations due to a long shadow period immediately preceding, the VAP Alignment was a limited case involving only two (2) spacecraft and a subset of instruments.

Following the post-shadow season, the pressing need to bring one (1) observatory to full operational readiness drove the planning. Once each sensor was activated at full level on MMS-1, FPI.010.2 was executed via ATS, followed by fulltime pseudo-nominal operations. Pseudo-nominal referred to the fact that interference observations were still possible and the SITL process, while not in full operation, was able to be integrated in gracefully incremented steps. Each successive observatory was brought up in that manner, and the SITL process evolved, preparing for the four (4) observatory fleet.

With four (4) observatory SITL processing underway, the team was able to execute specialized tests for crosscalibration and interference identification. Following this cross-calibration campaign, the fleet went into full SITL operations, allowing the complete science team to participate and to debug the SITL process (which is intended to enable quick turn-around on science meta-data evaluation and to guide the science selections in the ROI of the previous orbit.) The science team also spent the last two (2) weeks prior to the transition to Phase 1 preparing their instruments by generating final software loads and tuning parameters. In this manner, the operational paradigm grew incrementally over the last several months of commissioning, providing science team engagement and education in preparation for Phase 1 operations.

Towards the end of commissioning a transitional period was planned to hand over day-to-day operations from the ITFs and ISSEs to the POC. Although the ITFs continued to participate by defining activity requests, many of these requests became sufficiently repetitive that they no longer required ITF participation in their execution. In contrast, major activities such as updates to flight software or operational configurations continue to include ITF participation.

One significant exception to ITF operation is the routine update of CIDP command sequences. The CIDP includes a capability for instruments to define command sequences that can be executed on board. CIDP command sequence updates will be required routinely (about monthly) throughout the mission. This would represent a significant cost burden if the ITFs, ISSEs, CIDP, and POC personnel were all required.

To minimize this involvement, a variety of tools were created to ease the operational burden on the POC such that participation from non-POC personnel was eliminated from most of the command sequence update process. The definition of command sequence updates are prepared by the ITFs and provided to the POC. The CIDP ITF produced 
a general-purpose tool to automatically generate the CSTOL upload products from the raw data provided by the ITFs. Together with the POC, the CIDP ITF generated a CIPD command sequence upload process that encapsulates the expert knowledge of CIDP personnel such that the POC can execute these same processes over and over without routine CIDP ITF or ISSE participation. Figure 6 shows the high level view of the upload process (note the complexity, the details are purposely obscured).

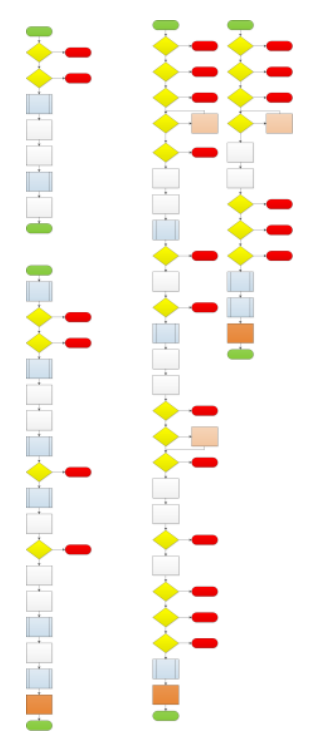

Figure 6. CIDP Command Sequence Upload Process (diagrammatic View)

The POC's steps have been minimized to the following:

1. Run the CSTOL generator tool against the raw command sequence files provided by the ITFs to produce the upload and commit CSTOL procedures for each observatory.

2. Execute the upload procedure on each observatory.

3. Execute the commit procedure on each observatory.

4. Execute the reconfigure procedure on each observatory.

By breaking the process into these large steps, recovery from difficulties is simpler. If the upload cannot be completed due to unexpected loss of signal, for example, a simple abort command can be issued for an in progress upload when contact is restored and the upload procedure can be rescheduled for a subsequent contact.

\section{LESSONS LEARNED}

\section{Four $S / C=$ Production}

The commissioning plan was developed on the presumption that each test would require the same estimated time duration, regardless of repetition, since many activities involved a long series of commanding or wait-states Given the constraints on each activity and the rules listed in Section 3, on paper, the commissioning schedule consumed virtually all the calendar time allotted. In practice, repeating an activity more than once allowed everyone to become more familiar with the process. By the third or fourth execution of a commissioning activity, the total time to complete the activity was reduced significantly. MMS had an additional benefit, as each S/C had multiple copies of several instruments or required multiple executions of the same procedure on the same component (e.g., ASPOC). This proved to be an amplifier for decreasing execution time, and provided plenty of opportunity to fine tune the procedures and eliminate unneeded or wasted effort and time. However, some activities grew in execution time and often the DSN contacts were shorter than the minimum requested. Therefore, having allocated the original time estimates for an activity for every instance provided the schedule with contingency opportunities. This method of developing the original schedule also provided opportunity for additional activities not previously considered. In general, commissioning was completed several weeks ahead of schedule. The lesson should be to budget and schedule for the worst case, but be prepared to take advantage of early completion to perform opportunistic activities and begin operation early. Given a fleet of uniform design with more than four $\mathrm{S} / \mathrm{C}$, planning for reduced commissioning time after the second or third run of an activity would be reasonable from a planning perspective without adding significant risk.

\section{"Test as you Fly"}

To the extent the program budget made possible, the MMS development team incorporated a "test as you fly" approach to operations. Early in I\&T a representative of the POC was embedded within the IS test team to provide operational experience and knowledge continuity. Significant effort also went into mission simulation operations, both nominal and anomalous, and every attempt was made to develop scripts for I\&T that could be easily transitioned to flight procedures. The team also performed testing from scattered locations in order to verify the dispersed communications protocols necessary for flight commissioning. The attempt to 'test as you fly' was generally successful, but there were a few places where the approach fell short. Both successes and shortcomings are described in the following paragraphs.

There were several successful aspects to the MMS team's "test as you fly implementation." Including POC employees on the I\&T team was very helpful in developing an institutional resource with IS operational experience, improving the quality of the scripts, and easing the transition from ground to flight procedures. In a like manner, the implementation of high quality mission simulations helped to prepare the commissioning team for the commissioning activities, the pace at which they were conducted, and the limits on the flexibility to rearrange activities. Of particular value was the launch and early orbit simulation, which included a number of simulated mission 
anomalies. This simulation gave a realistic appreciation of the "feel" of flight and allowed the teams to test their approach to anomaly resolution, resulting in useful changes to procedures as well as a more successful commissioning activity. The commissioning phase of the mission was materially improved by these aspects of "test as you fly" ground operations.

There were several areas in which the "test as you fly" preparation was inadequate. The most serious from a budget/schedule standpoint was the amount of effort necessary to translate ground scripts into flight procedures. The ITF I\&T effort used a ground software system called Ground Support Equipment Operating System (GSEOS), while the flight operational ground systems are the Operations and Science Instrument Support Command and Control (OASIS-CC) at the POC and the Advanced Spacecraft Integration and System Test (ASIST) at the MOC, which uses STOL. Addressing the differences between these ground and test systems had a non-zero cost. Both ground systems used by the instrument suite implemented a CSTOL variant, with the GSEOS implementation reasonably close to that of OASIS-CC. However, many of the I\&T scripts for the IS had not been structured in a way that met flight operational coding standards. This required that the I\&T scripts be rewritten for use in a flight environment. The lesson learned is that, if at all possible, the I\&T development should use the flight command and telemetry (C\&T) system as well as flight coding approaches. Even if a common C\&T system is not used, a flight-centric coding standard should be established and followed prior to beginning I\&T, and I\&T work products destined for operations should be reviewed by operations personnel as part of the routine development process for I\&T. If this is not done, then extra work will be required to transition I\&T scripts to flight.

The most serious area of "test as you fly" shortcomings from a technical standpoint was the inability to reproduce the CFDP response delays inherent in operation with DSN and the ground station. During I\&T, the CFDP files were closed generally within a $20-40$ seconds. In flight, they would remain open 3-10 minutes (not seen in ground testing) and this resulted in a number of operational challenges including unexpectedly high processor usage, delays in data transmission, and increasingly complicated ground based data management. The operational difficulties eventually culminated in a new software load. The lesson learned was to ensure that future ground systems incorporate timing based upon flight norms.

The final "test as you fly" lesson exposed shortcomings found in the configuration of the mission "FlatSats". There are three FlatSats available for mission use, one at NASA/GSFC, a second at CU/LASP, and a third more limited test bed for CIDP-centric testing. The NASA/GSFC FlatSat has a complete $\mathrm{S} / \mathrm{C}$ bus configuration including a communication card along with an engineering model (EM) CIDP and FPI IDPU (central processing unit) that connects to a ground segment telemetry system equivalent to the operational one, particularly as regards CFDP processing. However, instruments are computer-based simulations with moderate to low fidelity; they produce an appropriate volume of data, but the data lacks the periodicity, size, and internal structure of instrument produced data. The CU/LASP FlatSat has no S/C hardware but has an EM CIDP and a complete set of IS hardware with medium to high fidelity. These instrument simulators typically run their flight software, and thus produce data of much higher correspondence to operation, although a robust science simulation mechanism is typically not included. The software test bed for the IS consists of an EM CIDP and a special test equipment (STE) that simulates the instruments, $\mathrm{S} / \mathrm{C}$, and ground segment. Thus, there is no end-to-end, full fidelity FlatSat available. As a result, we must deal with a variety of "exceptions" when we test scripts, and are unable to fully ground test some aspects of system operation. The lesson learned is to generate a fully capable, high fidelity FlatSat for mission use.

\section{Complexity vs. Flexibility}

Commissioning a science observatory with one or two instruments is a complex and intricate task. Multiplying the number of observatories by four, and the number of instruments by 10 , gave MMS a level of complexity that had to be offset by other factors in order to commission successfully. The primary offsetting factor for MMS was flexibility: There were times when MMS had adequate flexibility resulting in simplification and efficiency; there were other times where the lack of flexibility resulted in significant inefficiency; and there were times when no amount of flexibility could have made it easier.

One area of great flexibility was provided by MMS instrument specific command panels. These command panels included a set of pre-defined and pre-validated operational procedures that were developed during the planning phase and expanded as the mission progressed to incorporated lessons learned. While most operational sequences were created uniquely and vetted well in advance ( 24 hours or more) of an observatory contact, there were occasions when quick changes were required (often within a contact.) The existence of the instrument command panels allowed quick processing of operational sequence changes, or entirely new sequences that could invoke the existing procedures found in the control panels. The flexibility provided by the captured operational experience contained in those panels thus mitigated the complexity of late breaking changes, minimizing impacts to the overall commissioning schedule.

The as-developed commissioning plan had a high level of complex integration. As long as all of the operations went well, and all of the development efforts for the succeeding operations were on time, the system worked. However, the complexity left no room for error. As expected, there were times when instrument anomalies or missed passes resulted in the need to rearrange the sequence of operations. Some 
rearrangement was as simple as swapping a sequence of operations between two observatories. Inevitably however, there were times when a RF contact could not be used for commissioning activity, either because of strict sequencing constraints or because a substitute procedure had not been developed. If a set of completed procedures had been ready for execution, and if the execution sequence order could have been made more flexible, a more efficient use of RF time could have been achieved. This is an example of a case where no compensating flexibility was available to offset the mission complexity. Fortunately, there was enough extra time in the commissioning schedule that the complexity caused no ultimate problem.

The MMS commissioning team discovered how truly complex MMS operations could be when a science opportunity arose towards the end of the eclipse season for a coordinated observing campaign with the Van Allen probe mission. (This opportunity was not included in the baseline commissioning plan.) It took a great deal of planning, procedure development, and engineering calculation to implement the joint campaign while ensuring that all of the instruments on the two observatories involved were ready to take quality science data and that the power margins were adequate. It also required developing the appropriate absolute time sequence (ATS) uploads on relatively short notice. The sheer complexity of the job, occurring relatively early in the mission, resulted in an extremely difficult period of effort for the POC, MOC, ITF, and ISSEs. The lesson is that, sometimes, operating a complex mission is going to be difficult no matter what compensating provisions are included.

\section{Commissioning Team Shift Scheduling}

Because the average length of an MMS orbit during Phase 0 was just under 24 hours, the commissioning teams had to choose between a shift schedule that followed a 24 hour day or one that defined the orbit as the standard day. Following a 24-hour day allowed crews a routine that they could plan for consistently throughout commissioning. The day shift crew could maintain a normal sleep-wake schedule and continue to interact with others in their organization during standard business hours. The night shift could adapt to a set of core work hours that wouldn't change over the course of the 6-month commissioning phase. Defining a day to follow the length of an orbit required crews to shift their sleep-wake times routinely and could cause confusion as to when shifts started on "transition" days. However there were clear benefits to activity execution in this mode.

The observatory commissioning team kept their shift times consistent throughout all of Phase 0. Although maneuvers required a small crew to support at any time of day or night, planning activities and Command Authorization Meetings (CAM) were performed during local day shift. Similarly, $\mathrm{S} / \mathrm{C}$ bus activities occurred at any time, were often passive activities, included little real time commanding, and were subject to offline data analysis. Because of this, there was no driving need for the observatory crew to follow the orbit.
The POC's decision to align crew shifts with the MMS orbit provided significant benefits to the IS commissioning plan with minimal impact to the crews themselves. While this approach was more challenging to the humans on shift the benefits to the overall commissioning flow far outweighed the inconveniences. The POC shift handovers occurred near perigee and apogee each orbit. Because of the ground contact schedule and the need to execute the majority of the IS commissioning activities over DSN sites, this schedule guaranteed that one handover per day would occur during a quiet part of the orbit. The POC understood that, with the command intense activities planned for all instruments, trying to fit in a handover briefing while executing a realtime activity would introduce many opportunities for error. The added distraction of translating a large amount of information to an incoming crew member could easily cause mistakes in communication on console or the overlooking of anomalous indications in telemetry.

Organizing crew shifts to follow the orbit allowed the POC to take full advantage of the gap in DSN contacts during that part of the orbit. Without DSN contacts to execute commissioning activities the POC, ITFs and ISSEs were not needed on console. The ISSEs also realized the advantages of this schedule and aligned their shift splits with apogee/perigee as well. This provided the ideal time to schedule the daily IS planning meeting and activity walkthroughs and ensured that all teams were able to participate. This did present some difficulties coordinating with the MOC mission planners as there was a great deal of overlap between the observatory Tactical and IS Planning meetings. ITFs and ISSEs were often on console during these meetings and couldn't always participate as fully as they wanted to. To bridge this gap one member of the POC planning team would attend the observatory Tactical meeting and serve as the voice of the IS when needed. As the local time of perigee evolved, the time of the POC Planning meeting and the Tactical Planning meeting began to align and the commissioning team combined these two meetings starting after the shadow season. This did still present attendance problems at times, but since the commissioning schedule post-shadow was more relaxed it was less of an issue to work around.

\section{Conclusions}

MMS was a complex and challenging mission to commission. With careful attention to detail in the commissioning planning process complex, multiobservatory missions can be successfully commissioned on schedule and within budget. To date, MMS successfully completed the variations in formation separation over the first two months of Phase 1A, settling on separation scale sizing for the remainder of the Phase. The mission continues to provide remarkable science results and has not encountered any major anomalies among the fleet. 


\section{ACKNOWLEDGEMENTS}

The authors wish to thank NASA Goddard for the opportunity to build this exciting fleet of observatories. We would also like to thank the entire MMS team involved in commissioning for their hard work and dedication that made MMS commissioning so successful and gave us many experiences we wanted to share. Finally, we would like to thank Martin Wasiewicz for his careful review of this paper.

\section{REFERENCES}

[1] Zell, Holly, Ed. Magnetospheric Multiscale. Oct. 18, 2015. Web. Oct. 20, 2015. http://www.nasa.gov/mission_pages/mms/index.html

[1] J.L. Burch, R.B. Torbert. The Magnetospheric Multiscale (MMS) Mission, Preface, Space Science Reviews (online), p. 1.

[2] J.L. Burch, T.E. Moore, R.B. Torbert, B.L. Giles. Magnetospheric Multiscale Overview and Science Objectives, Space Science Reviews (online), p. 2.

[3] Burch and Torbert, p.1.

[4] Burch, Moore, Torbert, and Giles, pp. 9-10

[5] LASP. CSTOL Reference Manual. Version 1.0. January $11,2010$.

[6] GSE Software, Inc. GSEOS 6.0 User Manual. GSE Software, Inc. 1998-2012. PDF file.

[7] Fung, Edwin, Ed. "Advanced Spacecraft Integration \& System Test Software (ASIST)". ASIST HOME. April 9, 2012. NASA. Web. Oct. 20, 2015. https://nasaasist.gsfc.nasa.gov/index.html. 


\section{BIOGRAPHY}

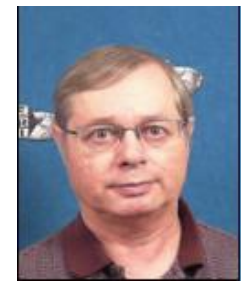

Paul Wood received a B.S. in Mathematics, Computer Science, and Systems Design from University of Texas at San Antonio, in 1979 and an M.S. from Purdue University in computer science in 1983. He has been with Southwest Research Institute for more than 30 years. He is the software lead for the MMS HPCA instrument, software lead for the MMS CIDP for the last two years, and is one of the IS systems engineers for MMS. A staff analyst with SwRI, he worked on the flight software for the Swift XRay Telescope and on various programs including robotics and telecommunications systems.

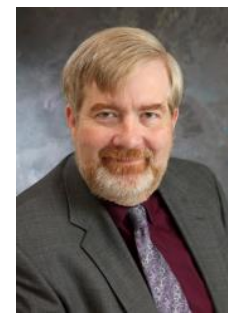

John Stone received a B.S. in Engineering Science (computer engineering concentration) from Trinity University in San Antonio and an M.S.E. with a concentration in Electrical Engineering at the University of Texas at Austin. He is the payload electrical systems engineer for MMS and is currently an Institute Engineer and chief engineer of the Space Systems Directorate at Southwest Research Institute (SwRI.) He has worked at SwRI for 28 years and has broad experience in architecting, designing, building, testing, and operating space-flight data systems and instruments.

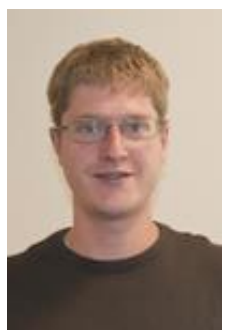

Patrick Smith received a B.S/M.S. in Aerospace Engineering from the University of Colorado in 2005. He has been working with the Mission Operations and Data Systems group at the Laboratory for Atmospheric Space Physics (LASP) for 10 years, giving him the opportunity to support operations on many programs, including SNOE, QuikSCAT, ICESat, SORCE, AIM, SDO EVE, and GOES-R EXIS. He has worked as the ICESat Flight Dynamics System Operations and Planning lead and was the MMS IS Commissioning Lead. He is currently working as the MMS POC Flight Director.

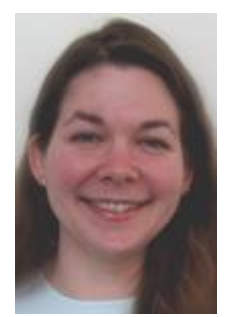

Jennifer Reiter received a B.S. in Engineering Physics from the Colorado School of Mines. She has been with the Mission Operations and Data Systems group at Laboratory for Atmospheric and Space Physics for 8.5 years and previously worked in Integration and Test at Lockheed Martin Space Systems. She has worked as an operator and planner for the ICESat, QuikSCAT, SORCE, AIM, Kepler, and MMS missions at LASP and is the Lead Trainer for LASP's Mission Operations group. Jennifer is currently part of the POC Planning team for MMS.

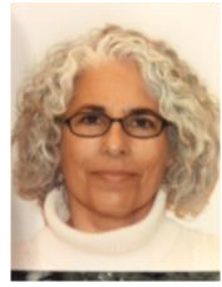

Cheryl Gramling received a B.S. in Aerospace Engineering from University of Maryland, currently with over 30 years at NASA/Goddard Space Flight Center. She was the flight dynamics lead for MMS until 2010 when she became the Commissioning Lead. She has served as the navigation and mission design lead for several missions and is currently the Center's Senior Navigation Systems engineer working on advanced onboard navigation and communication systems 